AIAA 2002-1355

\title{
EVACUATED ENCLOSURE MOUNTED ACOUSTIC ACTUATOR AND PASSIVE ATTENUATOR
}

\author{
Benjamin K. Henderson ${ }^{1}$, Steven A. Lane ${ }^{2}$ \\ Air Force Research Laboratory/VSSV, Kirtland AFB, NM 87117-5776 \\ Steven F. Griffin ${ }^{3}$ \\ Boeing-SVS, 4411 The 25 Way, Suite 350, Albuquerque, NM 87109 \\ Joel Gussy ${ }^{4}$ \\ University of Michigan, 3055 Whisperwood Dr, Ann Arbor, MI 48105 \\ Kevin M. Farinholt ${ }^{5}$ \\ Virginia Tech Mech. Eng. Dept., 307 New Engineering Bldg., Blacksburg, VA 24061-0261
}

\begin{abstract}
It is often desired that acoustic actuators or passive attenuators be mounted in relatively small, sealed enclosures to meet space constraints or in order to make the device unobtrusive. However, the large stiffness contribution from the air volume of such an enclosure can significantly increase the natural frequency of a conventional system, degrading performance at low frequencies. In this work, a novel concept is presented in which a diaphragm is mounted in a small, sealed enclosure, yet the overall system exhibits an extremely low natural frequency. This is accomplished by partially or completely evacuating the air from the enclosure to minimize the air-spring effect. A buckling suspension, exhibiting a nonlinear spring-rate, is utilized to counteract the large loads associated with the pressure difference across the diaphragm while retaining a low stiffness in the range of operation. This work presents theory, modeling, simulations, and experimental results from a prototype that demonstrates the utility of the proposed concept.
\end{abstract}

\section{INTRODUCTION}

The structural dynamics of enclosed volumes can generate an acoustic response that, through structuralacoustic interaction, can damage a delicate payload in a

\footnotetext{
${ }^{1}$ Research Aerospace Engineer, AFRL/VSSV, Senior Member AIAA

${ }^{2}$ Research Aerospace Engineer, AFRL/VSSV, Non-member AIAA

${ }^{3}$ Senior Engineer, Boeing-SVS, Senior Member AIAA

${ }^{4}$ Student, Michigan University, Member AIAA

${ }^{5}$ Student, Virginia Tech, Non-member AIAA
}

commercial space launch. Proposed approaches to mitigate this acoustic environment often involve the use of acoustic actuators, such as acoustic actuators (loudspeakers), or passive attenuator elements. Such elements are commonly mounted in sealed enclosures, or cabinets, to enhance acoustic radiation by preventing dipole behavior. However, the mechanical resonance frequency (i.e. the natural frequency) of the speaker may be significantly increased due to the air-spring effect of the air volume within the enclosure. This degrades the low-frequency performance of the speaker, since speakers radiate sound less efficiently below their natural frequency. Therefore, speakers designed for low-frequency applications are often mounted in large enclosures. Large-volume enclosures have less of an air-spring effect, which allows for a lower resonance. Although this is acceptable for most applications, many applications have constraints on the amount of space available for the speaker enclosures. Examples include compact sub-woofer designs, and active noise control actuators for automobile, aircraft, and launch vehicles. In such cases, it is desired to have speakers mounted in small enclosures that maintain good low-frequency performance. 
If an active feedback circuit is implemented to regulate the motion of the diaphragm, the loudspeaker's natural frequency can be tuned to a specific frequency. Such feedback loops have been widely used to control diaphragm motion and produce speaker systems with enhanced low-frequency performance ${ }^{1,2}$. In addition to audio applications, motion controlled speakers have also been successfully implemented in noise control applications where the low frequency response of the actuator is crucial ${ }^{3,4}$. However, this technique has the disadvantage of requiring active feedback circuitry and additional power sources, and may not be practical to implement in volume and mass critical aerospace applications.

The concept introduced here uses a novel approach to develop an acoustic actuator or passive diaphragm mounted in a small sealed enclosure that exhibits a low natural frequency. This is accomplished in part by partially or completely evacuating the air from the enclosure to minimize the air-spring effect. The conventional diaphragm suspension is replaced with a buckling suspension system that produces a non-linear spring-rate (stiffness). The suspension is designed to support the large loads associated with the pressure difference across the diaphragm while simultaneously exhibiting a low stiffness on the order of conventional speaker suspensions.

In the following, an analysis of the design concept is presented, and a rudimentary model is developed. Numerical simulations are presented to demonstrate the advantages of this approach using parameters of a typical loudspeaker. This is followed by experimental measurements of a proof-of-concept prototype device that validate the given models and theoretical predictions.

\section{THEORY}

To illustrate the proposed concept, a coupled model of the electro-mechanical speaker dynamics and enclosure will be presented. Then the effects of implementing a non-linear suspension and evacuating the enclosure will be discussed. The system will then be modeled as a baffled piston, and the effects of the design parameters on the on-axis radiated sound pressure will be computed to illustrate the design advantages.

A schematic diagram of a typical moving-coil loudspeaker is presented in Figure 1. The diaphragm is attached to the frame by the spider and the surround, both of which provide stiffness and damping to the loudspeaker system. At low frequency, the speaker responds as a simple mass-spring-damper system, and can be modeled as shown in Figure 2 using a mass element representing the moving mass of the system, a spring element and a damper element, representing the stiffness and damping of the speaker suspension, respectively, and an electromechanical force input. The electrical dynamics of the loudspeaker can be modeled as shown in Figure 3 by an inductor, a resistor, and a controlled voltage source. The inductance effect results from the voice coil, and the resistor models the DC coil resistance. The voice coil is positioned in a magnetic field created by a permanent magnet. A voltage, $v_{a}(t)$, applied across the speaker input terminals pushes current through the voice coil. The passage of current through the coil wire creates the driving force that moves the attached diaphragm and generates the acoustic pressure response. The movement of the voice coil within the magnetic field induces the flow of current in the opposite direction, which creates a back e.m.f. (electromotive force), modeled here as an AC voltage source. The back e.m.f., $v_{b}(t)$, is proportional to the diaphragm velocity, $\dot{x}(t)$, by the actuator constant $\psi$.

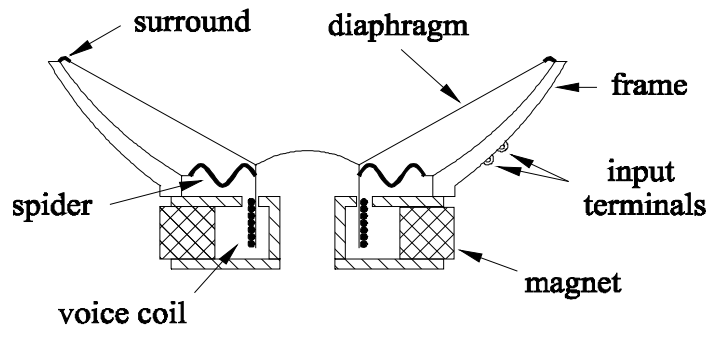

Figure 1: Schematic diagram of a typical moving-coil acoustic actuator

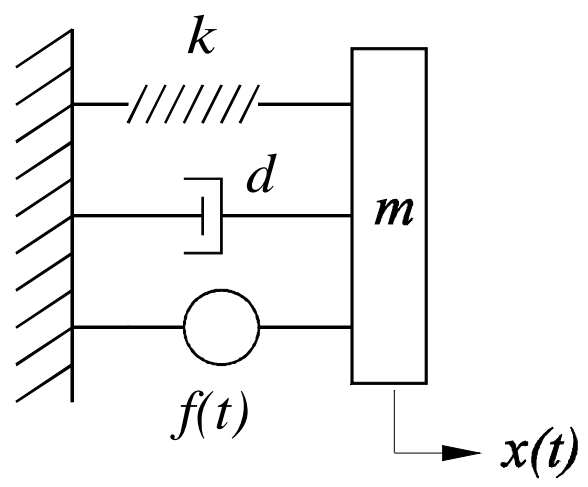

Figure 2: Mass-spring-damper model of the low frequency dynamics of a moving-coil acoustic actuator 


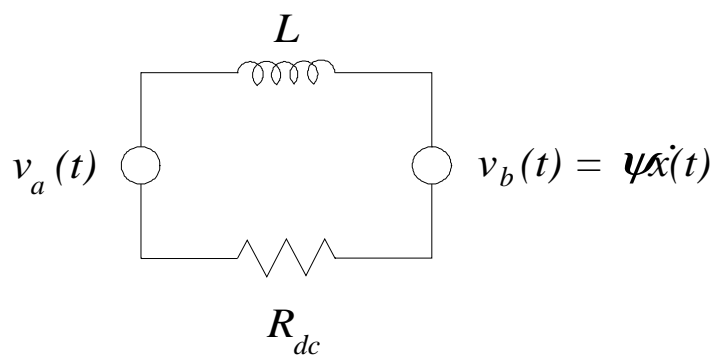

Figure 3: Model of the low frequency electrical dynamics of a moving-coil acoustic actuator

Using Newton's second law of motion, the mechanical dynamics can be modeled by a second-order differential equation, given as:

$$
m \ddot{x}(t)+d \dot{x}(t)+k x(t)=\psi i(t),
$$

where $i(t)$ is the driving current, $x(t)$ is the diaphragm displacement, $m$ is the moving mass, $d$ is the system damping, and $k$ is the stiffness. The electrical dynamics can be modeled by a first-order differential equation using Kirchoff's voltage law:

$$
L \frac{d i(t)}{d t}+R_{d c} i(t)+\psi \dot{x}(t)=v_{a}(t),
$$

where $L$ is the coil inductance, $R_{d c}$ is the DC coil resistance and $v_{a}(t)$ is the voltage applied to the terminals.

For the case of a sealed enclosure, the enclosed air volume behaves as an air spring in parallel to the suspension, and is simply added to the suspension stiffness, $k$. For an ideal gas under isothermal conditions, the air spring, denoted as $k_{a}$, may be represented by

$k_{a}=\beta A^{2}=\frac{\rho_{e} c^{2} A}{V}$,

where

$\beta \equiv \frac{\gamma P_{0}}{V}$,

where $A$ is the radiating area of the diaphragm, $\rho_{e}$ is the density of the air in the enclosure, $c$ is the speed of sound, $\gamma$ is the ratio of specific heat at constant pressure to specific heat at constant volume $(\gamma=1.4$ for air at standard temperature and pressure), $P_{0}$ is the static pressure of the enclosed air, and $V$ is the volume ${ }^{5}$. If the enclosure is small, the air-spring stiffness may greatly exceed the speaker suspension stiffness.
As evidenced by Eqs. (3) and (4), the spring constant may be reduced by decreasing the radiating area, increasing the enclosure volume, changing the enclosure gas, or reducing the gas pressure. Increasing the enclosure volume is the conventional solution, although it has the disadvantage that speaker enclosures tend to be very large when low-frequency response is required. The use of a gas other than air is problematic in that it requires that the manufacturer tightly seal the enclosure. Furthermore, the values of $\gamma$ for gasses that are both safe and readily available are fairly close to those of air, offering only a minimal advantage.

The other alternative, reducing the gas pressure, is difficult to achieve. As implied by the ideal gas law, pressure can be reduced significantly by maintaining a very low temperature, but this is a costly solution in terms of power and complexity. Assuming that the system is isothermal, the density of the air in the enclosure is directly proportional to the absolute pressure in the enclosure ${ }^{6}$. Therefore, a reduction in the internal pressure of the enclosure translates directly into a reduction in the stiffness of the air spring. This approach is more practical, and is used in the approach presented in this work.

The suspension for a conventional speaker diaphragm is relatively soft. As a consequence, even a small pressure difference between the front and rear of a conventional speaker cone would quickly "bottom-out" the speaker, preventing the cone from proper operation. Conversely, if the suspension were redesigned to have a large spring constant to counteract the load due to the pressure difference, it would have a very small excursion when driven at reasonable power levels, resulting in poor sound radiation. Additionally, the pressure difference would likely cause significant deformation or even failure of the diaphragm itself.

A nonlinear suspension is proposed to provide a solution to the pressure loading effect resulting from evacuating the enclosure. It is desired to design a suspension to carry the large static pressure load while exhibiting a reduced stiffness at the operating point. In the ideal case, the suspension stiffness is reduced to a very small value at the desired interior pressure level. If the enclosure were completely evacuated, the air-spring stiffness would disappear completely, and the residual stiffness would result only from the non-linear suspension element.

This desired nonlinear stiffness behavior has been observed in eccentrically shaped buckling beams ${ }^{7}$. Figure 4 presents a representative force versus deflection curve for a slender beam with an initial 
eccentricity. As the loading force increases to the desired load value, indicated as $F^{\prime}$, the diaphragm deflects from its initial position, $x_{o}$, to the desired operating point, $x$ '. The slope of the curve represents the spring-rate of the beam or support member. Notice that as the deflection increases, the slope of the curve, and hence the stiffness, decreases. This curve is valid as long as the material is not stressed beyond its elastic stress limit. It is desired to design a buckling support apparatus for the speaker system that behaves in this fashion. By careful design of the support apparatus, the stiffness can be made to approach zero at the operating point. This yields a system with very low stiffness contribution from the supporting apparatus. The damping of the buckling suspension can be optimized by the designer through material selection or passive damping treatments to achieve the desired performance.

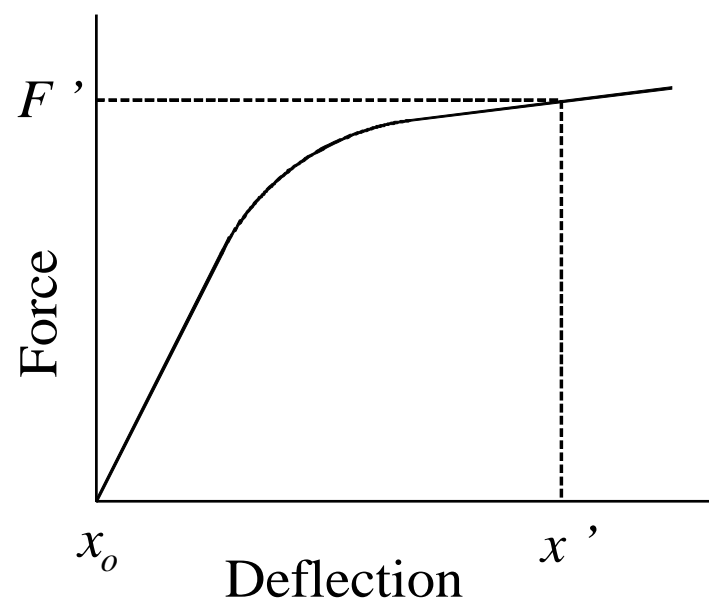

Figure 4: Typical force versus deflection curve for a buckling slender beam.

For simplicity, the enclosure mounted loudspeaker can be modeled as a baffled piston radiating into free-space. As such, the magnitude of the pressure response 1-meter directly in front of the speaker is given by

$$
\begin{gathered}
\text { pressure ampliude at } 1 \text { meter }= \\
2 \rho_{o} c|\dot{x}(t)|\left|\sin \left\{\frac{1}{2} \frac{\omega}{c}\left[\sqrt{1+a^{2}}-1\right]\right\}\right|,
\end{gathered}
$$

where $\rho_{o}$ is the air density, $c$ is the speed of sound, $\dot{x}(t)$ is the diaphragm velocity, $\omega$ is the frequency of oscillation of the diaphragm, and $a$ is the diaphragm radius ${ }^{5}$. This can be used as a reasonable approximation of the pressure radiated from a speaker mounted in a sealed enclosure as a function of the applied voltage. Together, Eqs. (1), (2), and (5) from a coupled model relating the applied voltage to the radiated sound pressure.

\section{SIMULATIONS}

Using parameters of a typical loudspeaker listed in Appendix A, three simulations were computed for various configurations of an enclosure mounted loudspeaker. In each case, the air-spring stiffness was computed using Eq. (3) and added to the value of $k$ given in the appendix. In the first case, the speaker was mounted in a $1-\mathrm{m}^{3}$ enclosure. This was contrasted to the response computed using a smaller, 0.0064- $\mathrm{m}^{3}$ enclosure. The frequency response functions relating the voltage input to the pressure output are given in Figure 5. These plots show that the smaller volume enclosure significantly increases the resonance frequency of the speaker, which reduces the acoustic output at low frequencies by approximately $15 \mathrm{~dB}$. The response using the $0.0064-\mathrm{m}^{3}$ enclosure system with a $90 \%$ vacuum is also shown in Figure 5. In this simulation, it was assumed that the suspension stiffness was reduced to $10 \%$ of the suspension stiffness given in Appendix A, and that the damping of the non-linear suspension is reduced by $50 \%$. In this case, the resonance frequency was reduced below the frequency of the large enclosure system. The low frequency pressure response was increased by approximately 3 dB. Although this is an idealized simulation, it clearly illustrates the possible advantages of the proposed concept.

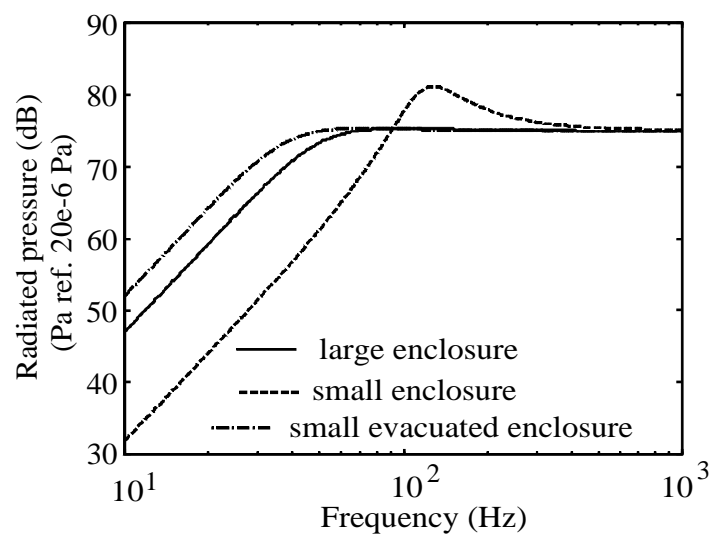

Figure 5: On-axis radiated pressure simulation results.

\section{DEVICE REALIZATIONS}

An active device can be built using a voice-coil and magnet apparatus in order to be used as an acoustic actuator. However, without the voice-coil and magnet, the device can be used as a passive acoustic attenuator. This is achieved by tuning the natural frequency of the device to couple to the acoustic modes of an enclosure. 
This realization allows the device to couple to and add damping to low-frequency acoustic modes.

Figure 6(a) is a schematic of a possible active realization of the device using a collapsible support mechanism. The support functions as the surround of a conventional speaker system by providing an airtight seal between diaphragm and enclosure. Figure 6(b) is an illustration of a device with a rectangular geometry.

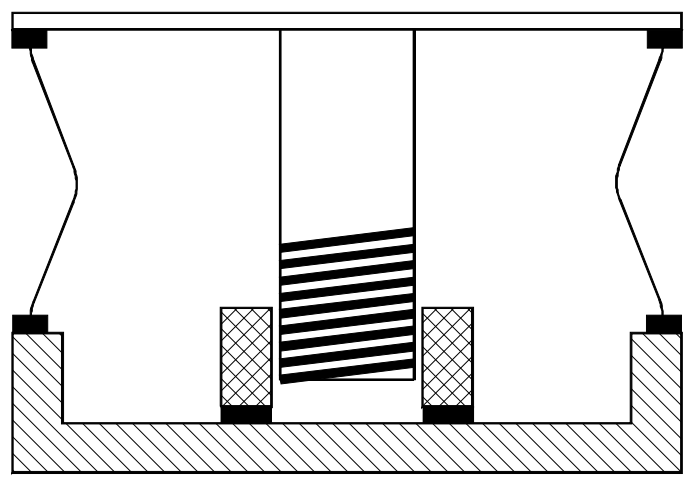

(a)

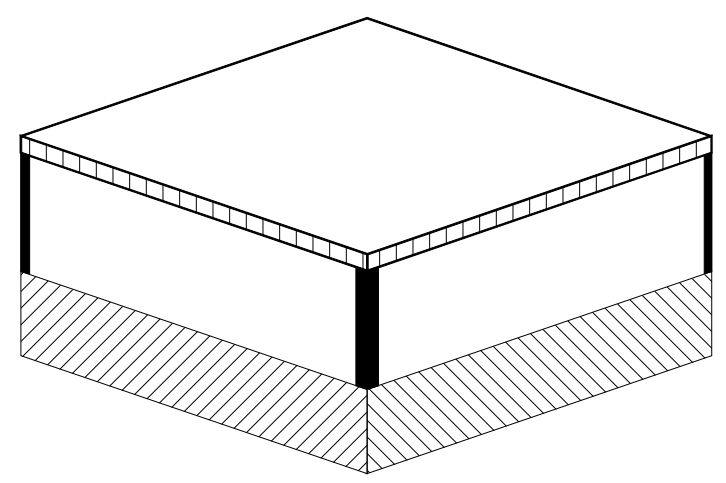

(b)

Figure 6: Schematic (a) and illustration (b) of the device using buckling beams to support the diaphragm.

\section{EXPERIMENTAL SETUP AND PROCEDURE}

A passive realization of the system shown in Fig. 6(b) was built and tested to experimentally investigate the effects of evacuating the enclosure and using a collapsible suspension. Figure 7 shows a photograph of the aluminum base, suspension system, and diaphragm. The interior dimensions of the aluminum base were $0.212-\mathrm{m} \times 0.212-\mathrm{m} \times 0.0415-\mathrm{m}$. Blue tempered springsteel shims replaced both the suspension and surround components that might be expected in a conventional speaker. The shims were affixed to the base and diaphragm with aluminum shims, which were attached with screws to provide a rigid, clamped condition. The diaphragm was an aluminum honeycomb structure sandwiched between two layers of carbon-epoxy composite. This material was chosen because of its low weight and high strength. Three threaded holes were drilled into one side of the aluminum base to accommodate a vacuum pump connection, a relief valve, and a pressure gage.

Early experiments showed that extremely thin suspension plates were required to provide the necessary deformation while avoiding plastic deformation and fatigue. As a result, each of the four suspension members consisted of three $0.127-\mathrm{mm}$ thick layers. The three layers were not bonded, and thus carried no shear loads at their interfaces. The deformable height of the suspension members was 55.7$\mathrm{mm}$, and each of the spring-steel members had a uniform initial pre-bend that resulted in a 4.7-mm initial out-of-plane displacement at mid-height. Such eccentricity allows for post-buckling behavior. The corners were covered with plastic patches that were bonded to the suspension with epoxy. Any leaks were sealed with silicone sealant to create a nearly air-tight enclosure. The total initial volume of the enclosure was $0.0048-\mathrm{m}^{3}$.

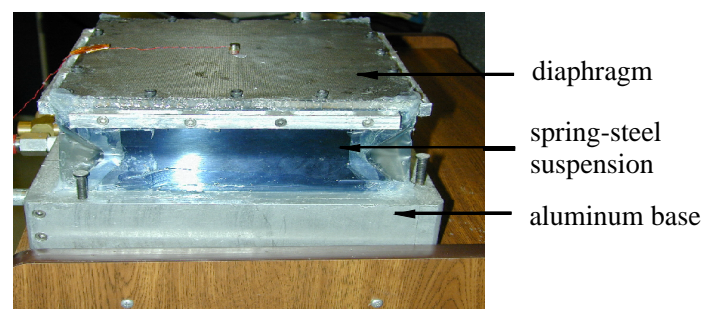

Figure 7: Photograph of the experimental prototype device.

The load curve of the suspension, from which stiffness is determined, was measured by applying a series of downward displacements through a load cell to the diaphragm and recording the resulting load cell voltage. The measurements were taken with the vacuum pump disconnected and the relief valve completely open to avoid any air-spring effects. In the next set of experiments, the change in the natural frequency of the system was then measured as a function of vacuum pressure. This was done using a vacuum pump to incrementally decrease the internal air pressure. At each increment, the natural frequency was measured using a force hammer, an accelerometer, and a spectrum analyzer. The accelerometer was affixed to the center of the diaphragm with wax, with its output connected to the spectrum analyzer. The force hammer (with a load cell) was connected to the secondary channel of the analyzer. The analyzer was set to record the transfer 
function between force hammer and accelerometer in the frequency domain, with the force hammer serving as a trigger signal. Ten signals were averaged and recorded, and the natural frequency was identified. This process was repeated over a range of vacuum pressures from $0 \mathrm{~Pa}$ to $13546 \mathrm{~Pa}$ in increments of 1935 $\mathrm{Pa}$.

The measured parameters of the system for the case of no vacuum and maximum vacuum were used to compute the effective baffled piston model parameters, which are given in Appendix B. The model was then used to predict the effect that the vacuum and buckling suspension would have on the radiated pressure of an active realization of the experimental device.

\section{EXPERIMENTAL RESULTS}

Figure 8 is a plot of the load-displacement curve for the experimental system. The slope of the curve at any point is a measure of the suspension stiffness at that displacement value. The suspension exhibits the behavior described with regard to Figure 4. That is, the stiffness is high at low displacements, such that a vacuum load might be supported, and low after the displacement exceeds a critical value. Therefore, the system would be expected to exhibit a low natural frequency in the range of operation.

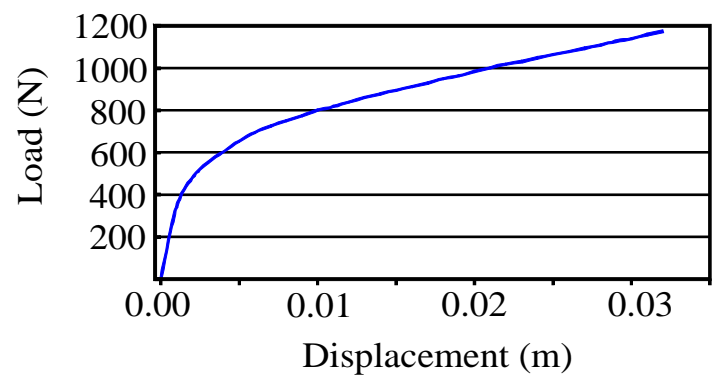

Figure 8: Load-displacement curve of the buckling suspension.

Figure 9 presents the experimentally measured natural frequencies of the prototype device for a range of vacuum pressures. As the enclosure is evacuated, the natural frequency of the device was reduced as predicted. The enclosure volume was also slightly reduced with increasing vacuum, since the diaphragm height was lowered. The resonance frequency of the system was reduced from $175 \mathrm{~Hz}$ to $59.5 \mathrm{~Hz}$ at the maximum vacuum pressure.

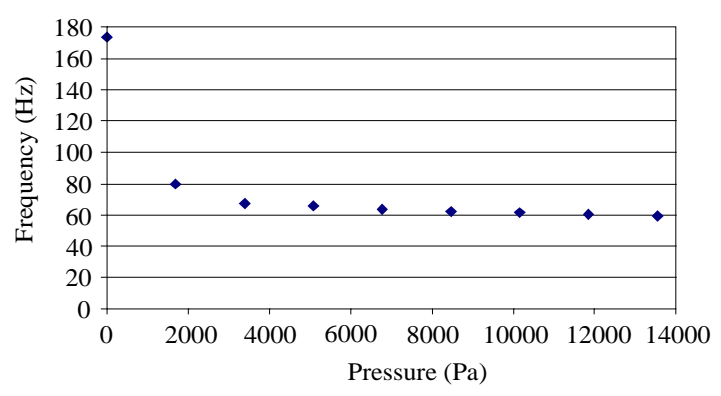

Figure 9: Natural frequency as a function of vacuum pressure for the experimental device.

Using the baffled piston model and the measured parameters of the experimental system given in Appendix B, the effect of the buckling suspension and the partial vacuum on the radiated pressure was computed and is presented in Figure 10. This simulation assumes that a voice-coil and magnet can be added to the system without having a significant effect on the measurements obtained from the passive experimental system. This is reasonable since the mass of a voice-coil is typically much less than the mass of the diaphragm used in this prototype. The damping ratio was estimated from transfer function measurements. The simulation predicts that the evacuated system would provide a significant increase (greater than $10 \mathrm{~dB}$ ) in the low frequency acoustic output of the system.

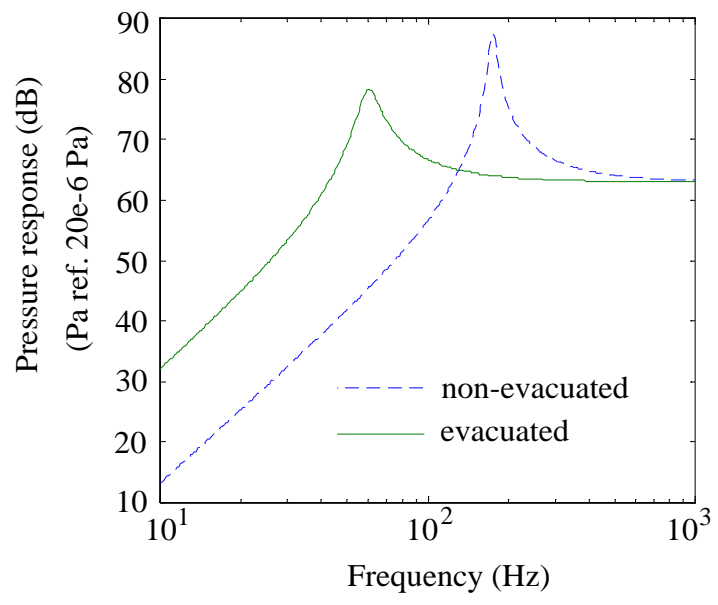

Figure 10: Predicted on-axis radiated pressure response using experimental values of the prototype device at full air pressure and when partially evacuated.

\section{CONCLUSION}

Simulations of a simple model of the proposed evacuated enclosure mounted speaker indicated that a significant increase in the low-frequency acoustic output may be achieved relative to conventional 
configurations. A prototype device was built and tested, and it was demonstrated that a non-linear suspension might be effectively used to support large pressure loads and yet provide low stiffness at the operating point. The results showed that partially evacuating the enclosure and using a non-linear suspension could significantly reduce the natural frequency of the prototype device. The performance of an active realization of the prototype device was predicted using a baffled piston approximation, and indicated an improvement in the low-frequency output of approximately $20 \mathrm{~dB}$. Although the prototype device is not practical for actual applications due to its size and mass, it does illustrate the key design innovations of this work.

\section{ACKNOWLEDGEMENTS}

This work was supported by the Air Force Research Laboratory, Space Vehicles Spacecraft Component Technology branch (AFRL/VSSV), and by the Air Force Office of Scientific Research (AFOSR) with Program Manager Dan Segalman.

\section{REFERENCES}

${ }^{1}$ Erath, L. (1992), Feedback System for a Sub-woofer Loudspeaker, US Patent \#5086473.

${ }^{2}$ Tanaka, S. (1996), Bass Reproduction Speaker System, US Patent \#5588065.

${ }^{3}$ Daniels, M. A. (1998), Loudspeaker Phase Distortion Control Using Velocity Feedback, US Patent \# 5771300.

${ }^{4}$ Lane, Steven A., Clark, Robert L., Southward, Stephen C., "Active Control of Low Frequency Modes in an Aircraft Fuselage Using Spatially Weighted Arrays," Journal of Vibrations and Acoustics, Vol. 122., No. 3, pp. 227-234, 2000.

${ }^{5}$ Kinsler, L. E. and Frey, A. R. (1982), Fundamentals of Acoustics, $3^{\text {rd }}$ edition, John Wiley and Sons, New York.

${ }^{6}$ Van Wylen, G. J. and Sonntag, R. E. (1986), Fundamentals of Classical Thermodynamics, $3^{\text {rd }}$ edition, John Wiley and Sons, New York.

${ }^{7}$ Seely, F. B. and Smith, J. O. (1959), Advanced Mechanics of Materials, $2^{\text {nd }}$ edition, John Wiley and Sons, New York.

\section{APPENDIX A}

Loudspeaker parameters used for Figure 5 simulations:

Moving mass, $m$ :

Force constant, $\psi$ :

$0.05 \mathrm{~kg}$

Stiffness, $k$ :

$6.7 \mathrm{~N} / \mathrm{A}$

DC coil resistance, $R_{d c}$ :

$3158 \mathrm{~N} / \mathrm{m}$

Damping constant, $d$ :

$4.7 \Omega$

Inductance, $L$ :

$7.5 \mathrm{~N} \cdot \mathrm{s} / \mathrm{m}$

Diaphragm radius, $a$ :

Free air resonance, $f_{s}$ :

$20 \mathrm{mH}$

$10 \mathrm{~cm}$

$40 \mathrm{~Hz}$

Enclosure parameters:

Large enclosure:

$1 \mathrm{~m}^{3}$

Small enclosure:

$0.0064 \mathrm{~m}^{3}$

Other parameters:

Sound speed, $c$ :

$343 \mathrm{~m} / \mathrm{s}$

Air density, $\rho_{e}$ :

$1.21 \mathrm{~kg} / \mathrm{m}^{3}$

\section{APPENDIX B}

Simulations parameters:

Moving mass, m:

$0.453 \mathrm{~kg}$

Force constant, $\psi$ :

$6.7 \mathrm{~N} / \mathrm{A}$

Damping ratio, $\zeta$ :

0.02

DC coil resistance, $R_{d c}$ :

$4.7 \Omega$

Diaphragm surface area:

$0.047 \mathrm{~m}^{2}$

Inductance, $L$ :

$20 \mathrm{mH}$

Enclosure volume without vacuum:

$0.0048 \mathrm{~m}^{3}$

Enclosure volume with partial vacuum:

$0.0032 \mathrm{~m}^{3}$

Natural frequency without vacuum:

$175 \mathrm{~Hz}$

Natural frequency with partial vacuum: $\quad 59.5 \mathrm{~Hz}$

\section{BIOGRAPHY}

Benjamin Kyle Henderson is the Manager of the Advanced Spacecraft Mechanisms Program in the Spacecraft Component Technology Branch at AFRL's Space Vehicle Directorate. He received his Ph.D. in Aerospace Engineering from Texas A\&M University in 2000, and has been a part of AFRL since 1994. He has worked in the areas of wave propagation, composites, acoustics, vibration isolation, and smart structures. 